\title{
La integración de los mercados en la sociedad: el caso de la transición económica rusa*1
}

\author{
Jorge Turmo Arnal \\ Universidad Autónoma de Madrid \\ e-mail: jorge.turmo@uam.es \\ Óscar Vara Crespo \\ Universidad Autónoma de Madrid \\ Ángel Rodríguez García-Brazales
}

Universidad Autónoma de Madrid

\begin{abstract}
RESUMEN
La transición de la economía colectivizada de la URSS a la economía de libre mercado de la Federación de Rusia en los años 90 presentó numerosos problemas. La gran reducción de la producción durante casi diez años, una elevadísima inflación y severos

1 Una versión preliminar del trabajo se presentó en el Tercer Simposio de la European Association of Evolutionary Political Economy, que tuvo lugar en Atenas en Septiembre de 2008. Los autores agradecen a los participantes en el Simposio las conversaciones mantenidas, entre ellas las de Alan Kirman que han permitido mejorar nuestro trabajo. Queremos agradecer muy especialmente los comentarios, sugerencias y críticas de Geoffrey Hodgson, quien se encargó de discutir nuestro artículo en la sesión correspondiente. Parte de sus sugerencias han sido incorporadas en el texto que presentamos. La responsabilidad por los errores y omisiones es enteramente nuestra.
\end{abstract}


problemas monetarios y cambiarios que desembocaron en la crisis de 1998 fueron el marco del proceso. Nosotros partimos del análisis de la realidad de la economía en la Unión Soviética y mostramos la influencia en los comportamientos de los agentes de su inserción en una sociedad en el que la libertad brillaba por su ausencia y la presión social era muy grande. Las redes creadas en época soviética tuvieron una influencia determinante en la forma en la que la transición económica se desarrolló. El proceso privatizador otorgó en gran medida la propiedad de las empresas a los antiguos dirigentes socialistas de las mismas. Su gestión se basó en las redes de contactos previas mucho más que en su reestructuración para atender las demandas del mercado. De esta forma configuraron la situación de la economía rusa en una vía que abocaba a la crisis que se consumó. Los mercados en Rusia surgieron mediatizados por el comportamiento de agentes concretos, insertos en unas dinámicas determinadas. Tenerlas en cuenta es la única vía para comprenderlas.

Palabras clave: Transición económica, Inserción ( Embeddedness) de la actividad económica en la sociedad, Proceso de privatización.

Códigos JEL: O5, N1.

\section{INTRODUCCIÓN}

En este artículo vamos a hacer una reflexión acerca del funcionamiento de los mercados y su integración en la sociedad a partir del análisis de la transición económica en Rusia, en un proceso que se extiende, según el consenso general de 1991 a 1998. El estudio de lo ocurrido durante ese proceso arroja mucha luz acerca del funcionamiento de los mercados cuando se tiene en cuenta su inserción en la sociedad, y no se da implícitamente por supuesto que su funcionamiento obedece a una lógica particular. La inserción ${ }^{2}$ del funcionamiento de los mercados en la sociedad permite comprender mejor porqué se dieron determinados procesos y los resultados de los mismos en términos de los grandes agregados macroeconómicos. Por esa misma razón se muestra muy útil para analizar el funcionamiento de los mercados con un carácter más general.

${ }^{2}$ El término inglés es «Embeddedness». La traducción directa es «Incrustación», pero preferimos hablar de «inserción» porque refleja mejor el concepto en español. Más adelante entramos en su definición. 
En el siguiente punto hacemos un breve repaso de los principales hechos y datos de la transición económica en Rusia y revisamos las principales teorías para explicar lo que sucedió durante esos años. En la tercera parte explicamos el enfoque de la integración de los mercados en la sociedad, tal y cómo ha sido desarrollado por varios autores, muy señaladamente por Granovetter, Etzioni, Abolafia y otros. En la siguiente sección analizamos el funcionamiento de los procesos de producción e intercambio en la economía soviética a grandes rasgos. La integración de esos procesos en la sociedad soviética fue muy fuerte y se mantuvo durante mucho tiempo, dando lugar a la aparición de una serie de dinámicas que persistieron una vez desaparecido el comunismo. En la cuarta parte dedicamos nuestra atención a los procesos de cambio que tuvieron lugar para transformar una economía centralizada en una economía de libre mercado. Una cosa es lo que se pensaba que era necesario hacer y otra, a veces muy distinta, lo que realmente se hizo. En la transición económica rusa era necesario crear los mercados porque, en un sentido estricto no existían previamente ${ }^{3}$, por lo que los procesos que tuvieron lugar son tan importantes, desde el punto de vista analítico, como los resultados de los mismos. Después explicamos cómo se comportaron los principales agentes de la economía rusa a lo largo de la transición, cómo resultaron afectados a la vez que influían en ella, de manera que el resultado final ha de tener en cuenta tanto los mercados como los agentes económicos. En la última parte ponemos de relieve que el análisis de la integración de los mercados en una sociedad concreta ayuda mucho a entender su dinámica y su funcionamiento.

\section{HeChOS Y DATOS DE LA TRANSICIÓN ECONÓMICA EN RUSIA}

Tras la disolución de la URSS en 1989, la nueva Federación de Rusia sufrió un período de intensa inestabilidad política que culminó en el fracasado golpe de estado de Agosto de 1991. Boris Yeltsin, presidente electo del país decidió a principios de Noviembre del mismo año poner en marcha las reformas necesarias para convertir a Rusia en una economía de mercado, abriendo así el período de la transición económica. Es difícil determinar en qué fecha el proceso termina. A lo largo de 1992 el proceso de

\footnotetext{
${ }^{3}$ Volveremos sobre esta cuestión más adelante. Según lo que se entienda, y defina, como mercado, en la economía soviética existían o no existían.
} 
liberalización de precios alcanzó a casi todos los sectores ${ }^{4}$. A finales de 1995 un gran porcentaje de las empresas estatales habían sido privatizadas y las grandes subastas para vender las acciones de Yukos, Sidanko y Surgutneftegaz ${ }^{5}$, a principios de 1997, suponen el final del proceso de privatización ${ }^{6}$. El proceso fue muy rápido y, en este sentido, los principales cambios ya se habían dado para esas fechas. Pero suele considerarse que la transición abarca hasta la crisis de 1998, su punto más bajo, y a partir del cual se inició la recuperación aumentando la producción y reduciendo la inflación y el déficit público, lo que conllevó una mejora del tipo de cambio del rublo. Sin duda, el posterior incremento en los precios de las materias primas energéticas que Rusia exporta colaboró decisivamente a ese cambio de tendencia. En este período vamos a centrarnos nosotros.

Lo más relevante del proceso fue la pretensión de crear una economía de mercado dónde antes no existía, de poner en marcha mercados y, además, conseguir que funcionasen. Se trata de dos procesos distintos, mucho más de lo que parece, que han de ser estudiados de forma separada. En otras situaciones de crisis económica, como la actual o reconstrucción de las economías de Japón y Alemania después de la Segunda Guerra Mundial, los mercados existían, aunque debilitados o seriamente dañados. Se trataba de reformarlos para conseguir que funcionen bien. En el caso de Rusia había que crear esos mercados y además conseguir que funcionasen correctamente. En términos estrictos, los mercados no existían en la Unión Soviética, no se daba la libre presencia de compradores y vendedores de bienes y servicios de forma continua intercambiando los mismos. En su lugar, la planificación soviética asignaba tanto los inputs de producción como los productos de la misma, decidía los precios a los cuales se habían de intercambiar (en muchos casos tan sólo se realizaba una imputación, no un pago como tal) y, lo que es más importante, decidía los bienes y servicios que había que proveer y en qué cantidades y cuáles no. Por consiguiente los retos a los que se enfrentaba la transición económica en Rusia eran mayores que la mera reforma de los mercados.

A este respecto cabe hacer una reflexión interesante. Los datos muestran que China ha tenido mucho más éxito en el proceso de transición que Rusia. Las tasas de crecimiento de su PIB, rondando o superando el $10 \%$ anual durante casi treinta años, le han

${ }^{4}$ Los precios de la electricidad el gas y el petróleo (para consumo interno) no se liberalizaron y en gran medida la situación sigue siendo la misma hoy en día.

${ }^{5}$ Shleifer, A., Treisman, D. (2000).

${ }^{6}$ Los sectores estratégicos, gas y electricidad, no se privatizaron, así como tampoco se hizo con la tierra, que siguió siendo propiedad colectiva. 
permitido mejorar su situación y el nivel de vida de los chinos de forma sustancial. Por eso se suele contraponer el caso Chino- una transición gradualista y exitosa- con el de Rusia - que durante el período que tratamos ha realizado una transición mucho más rápida con resultados negativos.

Pero desde el punto de vista «técnico» y entendiendo la transición como el proceso que lleva desde una economía centralizada a otra de libre mercado, el éxito corresponde a Rusia porque salvo los sectores de luz y gas y la tierra, el resto de la economía está liberalizado y en manos de empresas privadas. En China hay sectores enteros y provincias del país en las que la actividad económica sigue estando planificada en un 80$90 \%$, debido a que el gradualismo se ha aplicado desde el Este del país, la costa, hacia el Oeste, el interior. La economía china es mucho menos de mercado, en media, que la rusa, aunque la producción y el nivel de vida medios sean mucho mejores. Lo anterior pone de manifiesto que una transición económica puede ser más exitosa, las cifras cantan, aunque sea menos profunda, aunque el mercado se haya desarrollado menos que en otra. Es decir, que la mera liberalización de los intercambios y la privatización no asegura de por sí que los datos macroeconómicos sean buenos, que es el objetivo final. De esa manera podemos distinguir entre el proceso de paso de una economía centralizada a otra de mercado y el funcionamiento de esa economía de mercado.

Por tanto la transición económica en Rusia había de crear mercados para los bienes y servicios (incluyendo la tierra que no se privatizó), para el trabajo y mercados financieros en sentido general, debiendo el sector público reducir su dimensión a la propia de una economía de mercado. Además ese proceso tenía que ser un éxito. Cuando Yeltsin y sus asesores ${ }^{7}$ lo pusieron en marcha creían que era la única manera de detener y revertir el estancamiento y la debilidad de la economía rusa que databa de la época de Brezhnev y que no había sido atajado por la Perestroika de Gorbachov. Suponían que si el proceso era lento la resistencia de los agentes que tenían fuertes intereses en el mantenimiento de la planificación económica lo haría descarrilar, por lo que se decidieron por la vía rápida ${ }^{8}$, a pesar de que algunos optaban por una vía más gradual, siguiendo

${ }^{7}$ Por un lado estaban los asesores rusos que formaban parte de su gabinete. Entre ellos destacan Yegor Gaidar, Anatoly Chubais o Boris Fyodorov. Por otro lado los asesores extranjeros que tuvieron un papel más técnico.

${ }^{8}$ Los aspectos políticos de la fase inicial del proceso son cruciales. Las decisiones políticas, en las que pesaron decisivamente el riesgo de que se repitiese un intento de golpe de estado comunista como el de Agosto de 1991, marcaron el ritmo del proceso. La situación contrasta mucho con lo ocurrido en China. 
el ejemplo de Polonia9 . Desde fuera de Rusia algunos autores apuntaban que lo mejor era que el proceso fuese rápido basándose en razones teóricas ${ }^{10}$.

Como apunta Roland ${ }^{11}$ se daba por supuesto que la teoría económica que traían los asesores occidentales era lo mejor de que se podía disponer, sobre todo en lo referente al proceso de estabilización, que era lo que se adivinaba como punto clave de la transición. Ahora bien el mismo autor señala que cuando comenzó la transición no se sabía cómo privatizar una empresa socialista ni cómo conseguir que las empresas socialistas se ajustasen a las restricciones financieras propias de una economía de mercado ni, lo que es más importante, los efectos sobre la reasignación sectorial y la producción agregada de los distintos métodos de liberalización de una economía socialista. Un problema añadido era que, dados los previsibles problemas del proceso en su primera fase, se hacía necesario recurrir a los organismos internacionales, sobre todo el FMI, para obtener ayuda financiera. La contrapartida era la necesidad de obtener su acuerdo en cuanto a las medidas a adoptar.

Todo ello condujo a un conjunto de medidas que estaban basadas en lo que comúnmente se conoce como «Consenso de Washington», que incluían:

a) La liberalización de los precios.

b) Una política monetaria restrictiva y control del déficit público.

c) La privatización de las empresas estatales.

d) La reestructuración de la economía para mejorar la eficiencia agregada y la producción.

La primera y la tercera de esas medidas eran fundamentales para convertir una economía planificada y centralizada en una economía de mercado. Conseguir eso era equivalente a realizar la transición en términos técnicos, cambiar el marco institucional de la misma. Pero el cambio implicaba dos cosas: tomar las medidas necesarias, en términos legales, e implementarlas, lo cual a su vez necesita de la presencia de instituciones que se encarguen del proceso y que lo lleven a término. Un informe del Banco Europeo de Reconstrucción y Desarrollo (EBRD, 1999:26) explica con claridad que cuando hay una diferencia de ritmo entre los procesos -liberalización y privatización- y el desarrollo de las instituciones que han de hacerlos posibles la evolución de

\footnotetext{
${ }^{9}$ Dobrowski, M. (1997).

${ }^{10}$ Lipton, D. y Sachs, J. (1990).

${ }^{11}$ Roland, 2000:2.
} 
los mismos se resiente. Esa fue una de las cosas que ocurrieron en la transición económica rusa y que se puede entender mucho mejor si lo analizamos desde la perspectiva de la inserción de los procesos económicos en la realidad social como pondremos de manifiesto más adelante.

La segunda medida resultaba instrumental y de acompañamiento, puesto que era la única manera, si bien dolorosa, de evitar que el proceso de cambio condujese a un grave desajuste en la economía, una crisis inflacionaria y cambiaria, que fue lo que precisamente ocurrió en los años 1997-1998. El fracaso en este punto es a la vez una causa y una consecuencia de los problemas generales de la transición económica en Rusia debido a la forma en la que los agentes de la economía se insertaron en la nueva situación partiendo de sus formas de actuar en la época soviética. Como veremos, los agentes, incluso los que tenían que tomar las principales decisiones, no fueron inmunes a todo lo que había ocurrido en el pasado.

La última de las medidas era la fundamental. Ya durante la época soviética casi todos los analistas coincidían en afirmar que el problema de la baja productividad, de la deficiente información incorporada en los precios como señales y la falta de eficiencia derivada de un proceso de decisión enormemente centralizado eran las principales debilidades de la economía planificada ${ }^{12}$. Desde un punto de vista muy general y muy teórico se podía decir que la economía soviética estaba muy lejos de su Frontera de Posibilidades de Producción. Más allá de los aspectos distributivos, incluso para los mismos rusos el contraste entre la cantidad de bienes y servicios disponibles para la población rusa y los que se exhibían en los mercados de los países de economía de mercado de Europa Occidental era notable y desalentadora.

Se suponía que la liberalización y la privatización tan sólo podían traer consecuencias positivas para la economía en su conjunto. La liberalización de los precios permitiría dos cosas:

- Que funcionasen como señales informativas que ayudasen a las empresas y a los consumidores, sobre todo a los primeros, a tomar las decisiones de producción y consumo correctas, redirigiendo los recursos que se utilizaban en bienes y servicios sin demanda real hacia aquellos que sí tenían demanda y por tanto incrementaban la utilidad de los ciudadanos.

- Que las empresas se dedicasen a producir para aquellos mercados en los que hubiese demanda solvente.

${ }^{12}$ Para el estudio de la economía soviética remitimos a Nove (1969, 1977) o Dobb (1960) 
Este proceso por sí sólo implicaba una reasignación de factores de producción de unos sectores a otros, es decir una reestructuración de la producción, de forma que se pudiese producir más (reduciendo las ineficiencias previas) y más adecuado a las necesidades (utilidad) de los consumidores. Para hacerlo efectivo era preciso que pudiesen existir empresas privadas, por un lado, y que la gran mayoría de las empresas de titularidad pública se convirtiesen en privadas, por otro, de forma que pudiesen orientarse a las necesidades y demandas del mercado. Pero la reestructuración en su conjunto, que era lo fundamental, era tan sólo el resultado final de los procesos. El éxito de la reestructuración dependía crucialmente del buen desarrollo de los procesos de liberalización y privatización y del adecuado acompañamiento del control monetario y del déficit. Y nada de eso podía garantizarse a priori.

En gran medida puede decirse que había un consenso amplio acerca de los objetivos últimos de la transición económica en Rusia, así como en las medidas iniciales que había que adoptar. Pero no se sabía bien, ni entre los consejeros y ministros de Yeltsin ni entre los asesores internacionales, cuáles eran las estrategias concretas que era preciso adoptar para llevar a buen puerto la reforma. Desde nuestro punto de vista esta situación era un reflejo de la falta de conocimiento de cómo el funcionamiento de la economía en la Rusia soviética estaba insertada en la sociedad de ese momento. El análisis de los procesos abstractos de funcionamiento de una economía de mercado no puede ser el mismo que el de una economía centralizada no tanto por la naturaleza de una y otra, que son muy distintas, sino por la forma en la que están integradas en la sociedad. Quienes aconsejaron desde el exterior al gobierno de Yeltsin-Gaidar sobre las principales medidas a tomar y las estrategias a seguir conocían muy bien el funcionamiento de una economía occidenal y, de forma implícita, de qué manera la economía de mercado se inserta en la sociedad, aunque sea en términos generales. Pero no conocían, y les resultaba imposible hacerlo, cómo la economía planificada soviética se insertaba en la sociedad de su época y las consecuencias que tenía eso.

Los asesores rusos, que sí conocían a fondo la economía soviética ${ }^{13}$, sabían menos acerca del funcionamiento de la economía de mercado y de las transformaciones de

${ }^{13}$ Muchos de ellos eran altos funcionarios del PCUS y formaban parte del Staff económico del partido y del estado. Hasta finales de los años 80 eran convencidos socialistas y partidarios de la planificación. Tan sólo poco antes de la desaparición de la URSS se reciclaron en liberales y pasaron a considerar que todo el problema residía en convertir la economía planificada en una de libre mercado, y que para eso las recetas liberales eran suficientes. 
todo tipo, no tan sólo formales, que implicaba. Además consideraban que la manera en que los agentes económicos actuaban en la economía planificada cambiaría al pasar a una economía de mercado, porque el sistema de incentivos y de señales se modificaba drásticamente. Por tanto, y por la premura de tiempo en un proceso como el que estudiamos, su esfuerzo se dedicó a poner en marcha las reformas e ir resolviendo los problemas sobre la marcha.

Pasando a los datos, la evolución del PIB durante estos años es muy llamativa.

Tabla 1. PIB de Rusia

\begin{tabular}{|c|c|c|c|c|c|c|c|c|}
\hline 1992 & 1993 & 1994 & 1995 & 1996 & 1997 & 1998 & 1999 & 2000 \\
\hline 541,23 & 501,14 & 437,78 & 344,21 & 327,56 & 332 & 292,63 & 289,04 & 362,42 \\
\hline
\end{tabular}

Fuente: FMI. Billones de Rublos a precios constantes de 1990.

Los datos muestran que el PIB de Rusia casi se redujo a la mitad desde 1992 a 1999 y que la tendencia fue decreciente. La recuperación comenzó en 2000, pero el valor de 2002, diez años después del comienzo de la transición, estaba todavía por debajo del de 1992. A pesar de las críticas que estos datos han suscitado ${ }^{14}$, la tendencia es innegable: la economía rusa ha estado decreciendo durante ocho años. El PIB por persona era de $3900 \$$ en 1989 y $2240 \$$ en $1997^{15}$. El decrecimiento en el nivel de vida ha sido muy fuerte y los rusos se han empobrecido mucho. La transición económica no ha mejorado la vida de los rusos, el fin último de cualquier política económica.

Los efectos de esta reducción de la producción en el Mercado de trabajo son sorprendentes. La tasa de desempleo era del 5,2 en 1992 y creció los años siguientes hasta el 13,2 en 1998. Después disminuyó al 12,6\% en 1999 y 8,6\% en $2002^{16}$. Las hipótesis de que se iba a producir una recesión propia de la transformación económica implicaban una masiva transferencia de trabajadores desde las muy ineficientes empresas soviéticas, que no estaban orientadas hacia el mercado, hacia las mucho más eficientes

${ }^{14}$ Los críticos apuntan a que la economía informal creció mucho y que los datos oficiales no la tienen en cuenta. Las estimaciones basadas en el consumo de electricidad dan como resultado que la producción disminuyó un $27 \%$, pero levantan dudas porque el sistema soviético era muy intensivo en energía.

${ }^{15}$ World Bank, Milanovic 1999. Dólares constantes de 1995.

${ }^{16}$ Goskomstat of Russia. Russia in figures, Concise Statistical Handbook, Moscow 2003. 
empresas de la nueva economía rusa, regidas por criterios de mercado. Si ese hubiese sido el caso, el desempleo habría experimentado un gran incremento primero, conforme la producción de bienes «soviéticos» se reducía, para disminuir después a la par con el crecimiento de las nuevas empresas orientadas a las demandas de los consumidores. Pero los datos muestran que eso no ocurrió.

La inflación fue uno de los principales retos de la economía rusa desde los inicios de la transición. Debido a la liberalización de precios, se incrementaron más del $2000 \%$ en $1992^{17}$. Las cifras mejoraron después, pero en 1994 la inflación era todavía del 224\%. Los niveles de inflación están correlacionados con la política monetaria y el déficit fiscal del gobierno ruso en los 90. Durante muchos años no pudo recaudar impuestos suficientes para cubrir sus gastos. En 1992 el déficit del presupuesto era del $20 \%$ del $\mathrm{PIB}^{18}$ porque los gastos ascendían al 39\% del mismo mientras que los ingresos apenas alcanzaban el 19\%. Las cifras fueron disminuyendo pero en 1998 el déficit era todavía del 7,2\% del PIB. Durante estos años la monetización del déficit fue regla general y la alta inflación y la depreciación del rublo, el resultado del proceso.

Las cifras muestran con claridad que los resultados macroeconómicos de la transición rusa fueron muy decepcionantes a todos los niveles. Para analizar los procesos económicos que están detrás de ellos vamos a partir del estudio de la inserción de los mismos en la realidad social de la época soviética y los cambios que se dieron en estos procesos con la transición.

\section{LA INSERCIÓN DE LOS PROCESOS ECONÓMICOS EN LA SOCIEDAD}

Economic action is socially situated and cannot be explained by reference to individual motives alone. It is embedded in ongoing networks of personal relationships rather that being carried out by atomized actors (Swedberg y Granovetter 1992:9).

El enfoque de la inserción de los procesos económicos en la sociedad es una reacción ante lo que, desde la sociología ${ }^{19}$, se considera un excesivo atomismo de la teoría económica neoclásica. El problema, se arguye, es que el agente económico neoclásico

${ }^{17}$ Goskomstat. Russia in figures.

${ }^{18}$ Shleifer y Treisman ( 2000:43).

${ }^{19}$ El enfoque se sitúa dentro de la Socio-Economía, a la manera de los Essays in SocioEconomics de Amitai Etzioni. 
tiene un comportamiento sub-socializado, de forma que las preferencias que expresa y las acciones que realiza parecen no tener en cuenta para nada la sociedad en la que vive, que la estructura social no tiene influencia sobre sus comportamientos.

No entraremos aquí en la discusión acerca del planteamiento de la teoría económica neoclásica. Las ventajas, en términos de simplicidad y de tratabilidad de los problemas de un enfoque totalmente individualista, o atomista han permitido a la economía progresar en muchos campos. Al reducir el análisis a agentes individuales bien definidos, es posible analizar comportamientos y procesos a partir de la base de lo que cada uno de ellos hace. Además este tratamiento de los procesos económicos forma parte de la tradición científica de reducir los fenómenos a las unidades más pequeñas posibles para facilitar el análisis y poder después poder proceder a relacionar y agregar. Pero sí estamos de acuerdo en que determinados fenómenos se abordan mejor desde una perspectiva que tenga en cuenta que las decisiones de los agentes en el ámbito de lo económico no sólo no son independientes del marco social en el que se desenvuelven, sino que se explican mejor teniéndolo en cuenta.

Además en el caso de la transición económica en Rusia ese contexto social es todavía más importante. El Marxismo es un enfoque sobresocializado de la acción humana. El comportamiento de los agentes está determinado por su pertenencia a una clase social concreta y sus ideas y sistemas de valores no son el resultado de su propia elección, sino una consecuencia mecánica de la clase a la que pertenecen y de las relaciones de producción en las que están insertos. De esta manera el individuo no es auténticamente libre de elegir y es necesario romper esa determinación a través de la revolución socialista. La consecuencia de este planteamiento es que una sociedad socialista, como era la de la URSS antes de la transición económica, deja muy escaso margen de elección e incluso de configuración personal a los agentes. Determinadas preferencias y elecciones son severamente desalentadas, si no directamente penalizadas. Por tanto en este caso la inserción de los procesos económicos de los agentes en la sociedad en la que viven es mucho más fuerte que en una economía de mercado en la que existe la posibilidad de elegir y tomar decisiones.

En este punto estamos de acuerdo con Swedberg ${ }^{20}$ en que el enfoque de Polanyi ${ }^{21}$ no es correcto. No puede decirse que la integración de los procesos económicos en las estructuras sociales sólo se da en las civilizaciones preindustriales. La creciente e innegable importancia de lo económico después de la revolución industrial no implica que se convierta en un ámbito autónomo y autosuficiente, sino que las formas y maneras

\footnotetext{
${ }^{20}$ Swedberg y Granovetter 2001.

${ }^{21}$ Polanyi 1944 y 1957.
} 
en las que se produce esa inserción son diferentes. De la misma manera es diferente la manera en la que los procesos económicos se insertan en una sociedad socialista de lo que lo hacen en una sociedad de libre mercado, como después veremos, pero la inserción no desaparece por eso.

Desde un punto de vista general, Granovetter ${ }^{22}$ (2005:33) afirma:

Social structure, especially in the form of social networks, affects economic outcomes for three main reasons. First, social networks affect the flow and the quality of information. Much information is subtle, nuanced and difficult to verify, so actors do not believe impersonal sources and instead rely on people they know. Second, social networks are an important source of reward and punishment, since these are often magnified in their impact when coming from others personally known. Third, trust, by which I mean the confidence that others will do the «right» thing despite a clear balance of incentives to the contrary, emerges, if it does, in the context of a social network.

En la medida en la que todos estos procesos, o parte de ellos, tienen lugar, los comportamientos de los agentes económicos y por tanto los resultados agregados de los mismos resultan afectados porque están insertos en esa realidad social. En el caso de la Rusia soviética pondremos de manifiesto las enormes dificultades de los flujos de información, que están en la base de algunos de los más conocidos defectos de funcionamiento de una economía socialista. En cuanto a los premios y castigos no hay que insistir en que la sociedad soviética estaba, por principio, muy controlada y que existía todo un catálogo, a medias implícito y a medias explícito de lo que era correcto o no y lo que debía o no hacerse, de forma explícita para la actividad económica. En el caso de la realidad económica rusa las cosas son algo diferentes pero no puede suponerse que de la noche a la mañana las redes creadas en la época soviética desapareciesen, salvo un cambio radical en los comportamientos de los agentes.

Esa inserción de los procesos económicos en la realidad social era más fuerte en época soviética precisamente por la inexistencia de mercados libres. El espacio de libertad y de decisión que representan en su ámbito los mercados no existía en la URSS, debido a la radical desconfianza hacia ellos por razones ideológicas. En su lugar los procesos económicos se desarrollaban a través de un conjunto de instituciones y reglas. En ese sentido, los procesos económicos estaban mucho más insertos en la realidad social, apenas tenían autonomía alguna. Por poner un ejemplo conocido, las decisiones

${ }^{22}$ Granovetter, M. (2005). 
de consumo de los agentes se daban sobre un conjunto predeterminado de bienes y servicios cuyos elementos habían sido decididos por las autoridades encargadas de la producción. Los consumidores no podían de ninguna manera hacer valer sus gustos y preferencias puesto que las pocas señales que podían emitir, en forma de largas colas ante los mostradores de los productos más deseados, eran sistemáticamente desoídas.

Lo que se podía consumir era fruto de una decisión social (aunque no de una red, sino de un decisor anónimo y lejano) y el papel de las preferencias individuales era bastante reducido. Además se consideraba que el consumo era un «vicio» típicamente capitalista y por tanto eso afectaba a las elecciones de los agentes en la economía soviética. Hay que tener en cuenta que una sociedad de este tipo presenta un conjunto bastante extenso de redes que permean toda la vida cotidiana de las personas ${ }^{23}$. Es lo que Granovetter ${ }^{24}$ denomina «Relational Embeddedness». Los efectos de ese tipo de inserción dependen críticamente de la densidad de la red y de las relaciones, y en el caso que estudiamos era muy fuerte y se extendía a mucho más ámbitos además del caso que hemos traído a colación aquí.

El nivel más agregado de la inserción son las instituciones que surgen como consecuencia de ello ${ }^{25}$. Para Granovetter el proceso comienza en los niveles más básicos y, si continua, llega al nivel más elevado, que es la institución. La precondición para la aparición de las instituciones es la densidad y permanencia en el tiempo de las redes y sus relaciones. Desde este punto de vista la economía soviética, y la sociedad en su conjunto es un caso extremo de existencia y actividad de instituciones, y por tanto de redes muy densas. Pero el proceso no comenzó desde abajo hacia arriba, como sugiere Granovetter, sino al revés. La economía soviética no es el resultado de la consolidación de un conjunto de redes y su reificación en forma de instituciones, sino la deliberada construcción de instituciones cuyo objetivo era, en lo que hace a la economía, sustituir el funcionamiento de los mercados impidiendo las que desde el punto de vista marxista eran sus perversiones fundamentales.

Para conseguir sus objetivos era necesario que esas instituciones llegasen hasta el último rincón y el último agente de la economía por lo que ejercían una enorme presión sobre el comportamiento social y económico de todos ellos. El grado de instituciona-

${ }^{23}$ El PCUS puede entenderse desde esta perspectiva como una enorme red extendida por todo el tejido social del país con una estructura muy densa. Los objetivos del PCUS iban más allá de lo político y afectaban incluso a los usos de la vida diaria, entre ellos los procesos económicos de los agentes.

${ }^{24}$ Granovetter 1990:99.

${ }^{25}$ Granovetter 1990:102. 
lización de la economía soviética era un correlato del nivel de sobresocialización de los comportamientos de los agentes. En muchas ocasiones el comportamiento de los agentes en los procesos económicos en la Rusia soviética tan sólo podía explicarse por la enorme influencia de esas redes institucionales de las que continuamente emanaban normas de conducta y guías de comportamiento. En estas circunstancias resulta difícil suponer que las elecciones de los rusos en el terreno económico no resultasen influidas por el contexto social en el que vivían. El objetivo del PCUS y todas las demás redes conexas, era precisamente influir de forma determinante en el comportamiento de los rusos en todos los campos, incluido el económico para que se adecuase al ideal que se preconizaba. Pasamos ahora a analizar de forma breve los principales rasgos del comportamiento de los agentes en el sistema económico soviético.

\section{LA ACTIVIDAD ECONÓMICA EN LA SOCIEDAD SOVIÉTICA}

El funcionamiento de la economía soviética es sobradamente conocido y no lo vamos a detallar aquí. Los rasgos generales pueden consultase en Dobb (1960), Nove (1969 y 1977), Gregory y Stuart (2001) o Lane (1985). Nuestro interés se centra en el análisis de la forma en la que los comportamientos de los agentes económicos estaban influenciados por la sociedad en la que vivían.

Los consumidores en la sociedad soviética no estaban acostumbrados a disponer de bienes de calidad ni de variedad de bienes. La diversidad de color, tamaño o forma era muy reducida y muchos bienes producidos eran inservibles en la práctica. El conjunto de elección entre el cuál podían decidir era muy reducido. Sus preferencias no eran tenidas en cuenta y el acto de elección como tal era mucho menos usual que en las economías de mercado. Eso no implica que no tuviesen preferencias, sino que, a lo largo de muchos años, no pudieron ponerlas en práctica, se acostumbraron a elegir entre la muy reducida variación existente lo que en algunos casos limitaba la elección a comprar (lo que hubiese) o no comprar.

Los precios no reflejaban la escasez relativa de los bienes y servicios, dado que estaban fijados y controlados. Por eso, los precios incorporaban mucha menos información de la que incorporan en una economía de mercado. En realidad eran precios ficticios. Por tanto los consumidores soviéticos apenas prestaban atención a los precios, dado que además no había alternativas, sino a las cantidades de los bienes. En términos generales, los consumidores soviéticos se encontraban en la extraña situación, para un occidental, de que no podían maximizar su utilidad no por la restricción de la renta y 
los niveles de precios, sino por la no disponibilidad de bienes y servicios en los cuales gastar su reducida renta a los precios fijados. Un rasgo general de la economía soviética es el ahorro de las familias. Ese ahorro en cierto modo era forzado dada la no disponibilidad de bienes y servicios. La «elección racional del consumidor» tal y como la entendemos nosotros, apenas se daba.

Las empresas ${ }^{26}$ tenían como objetivo marcado por la planificación producir cantidades concretas de productos concretos. Había otros objetivos pero ese era el fundamental y sobre el que se basaba el sistema de incentivos y sanciones ${ }^{27}$. El sistema soviético pretendía controlar todo el proceso productivo y para ello el procedimiento más fácil era asignar en cada escalón las responsabilidades de forma individual y determinada para que no pudiese diluirse. Por tanto en una empresa el único responsable, y también quien disponía de un amplio poder discrecional era el principal directivo, el mánager ${ }^{28}$. Los directivos habían de asegurar el cumplimiento cuantitativo del plan, pero no habían de ocuparse en absoluto de aspectos cómo: los destinatarios del producto (lo decidía el plan), la financiación (venía del Banco Estatal de acuerdo con el plan), los trabajadores (asignados por la planificación), y desde luego cosas como el marketing o lo que denominaríamos desarrollo de negocio. Por tanto desarrollaron competencias en esos aspectos, pero no sabían nada acerca de todos los demás ni estaban interesados en ello. Cómo veremos en la siguiente parte estos managers desempeñaron un papel crucial en las empresas privatizadas durante la transición económica.

Sus problemas principales eran dos: por un lado asegurarse un suministro suficiente de equipo de capital y de mano de obra. Para conseguirlo inflaban de forma sistemática sus necesidades de equipo de capital y dado que existía una clara asimetría de información entre ellos y los planificadores, casi siempre lo conseguían. El problema con los trabajadores era diferente porque el desempleo no podía existir en un sistema socialista por lo que todos los trabajadores eran asignados a un puesto u otro. Pero por la misma

${ }^{26}$ Las denominaremos así aunque carecían de varios de los atributos de una empresa tal y cómo la concebimos en una economía de mercado. Eran más bien unidades de producción. Véase Granick (1954).

${ }^{27}$ El no cumplimiento del objetivo de producción acarreaba una penalización severa y no económica, sino administrativa e incluso penal (Nove, 1977). La presión y los incentivos negativos eran muy intensos y también de esta forma el comportamiento de los agentes concernidos, sobre todo los managers de las empresas, estaba claramente influenciado.

${ }^{28}$ Granick, D. (1960). 
razón no podían ser despedidos, sólo transferidos a otra unidad de producción. En general había trabajadores de sobra, pero poco productivos.

La clave de la producción eran los suministros. Dado lo estricto del plan era imprescindible disponer de la cantidad y calidad de los suministros necesarios en el momento adecuado. Se suponía que la planificación había de asegurar eso, pero en la práctica no lo hacía salvo en los sectores considerados prioritarios, como el material para Defensa. Era moneda corriente que los suministros llegasen en las cantidades requeridas pero tan mal manufacturados que en la práctica eran inservibles. Además el problema de los plazos era muy generalizado, en gran medida debido a las debilidades del sistema de distribución y los cuellos de botella que se generaban. En términos generales la economía de la URSS era una economía de vendedores y el lado de la demanda era mucho más débil. Dado que no existía un sistema de señales, vía precios, que transmitiese la información de la demanda a los productores el lado de la demanda, en este caso la de suministros, resultaba siempre perjudicado. La sobrecapitalización y el excesivo número de trabajadores en las empresas permitía en parte paliar eso puesto que, en caso de necesidad, se podía incrementar mucho la utilización de la capacidad productiva. Pero no era suficiente si los suministros no llegaban.

Para eso las empresas crearon la figura del tolkach ${ }^{29}$, un conjunto de personas que tenían, o creaban, una serie de contactos en otras empresas y que, a través de esa red aseguraban la llegada de los suministros. Los conseguidores crearon una red de intercambios informales que permitían mejoras paretianas al poner en contacto empresas con necesidades y posibilidades diferentes. Las empresas no podían vender sus productos más allá de sus contratos o fuera de los mismos, a pesar de que otras empresas lo necesitasen. La solución era el trueque. Las autoridades eran conscientes del funcionamiento de esa economía de trueque pero no podían detenerla sin perjudicar a las empresas. Hasta cierto punto los conseguidores y el trueque llegaban allá dónde el plan no podía, pero al mismo tiempo lo minaban. De esa forma se generaba una red muy extensa de contactos entre los directivos de las empresas, los conseguidores e incluso en algunos casos las autoridades locales y regionales que estaban interesadas en que las empresas de sus territorios cumpliesen con los objetivos. Los managers de las empresas se acostumbraron a actuar teniendo en cuenta esa red, la Blat y contando con ella ${ }^{30}$. Una actividad económica informal se desarrolló junto a la economía colectivizada formal.

\footnotetext{
${ }^{29}$ Berliner (1957) La traducción es la de «conseguidor».

${ }^{30}$ Un buen análisis del funcionamiento de esa red es el de Ledeneva (1998).
} 
Los trabajadores vivían en un entorno de pocos incentivos y poca presión. El mercado de trabajo no existía como tal ${ }^{31}$. Los trabajadores no corrían el riesgo de perder su trabajo o ser reemplazados, sino como máximo podían ser enviados a otra empresa. Por otro lado los incentivos eran muy reducidos. Se suponía que el trabajador soviético había de tener un alto nivel de auto-motivación porque la producción agregada se dividía entre toda la población de una forma muy igualitaria. En la práctica el nivel era muy bajo, y había que lanzar campañas, como la del Stajanovismo, para movilizar los esfuerzos de los trabajadores ${ }^{32}$.

Los incentivos materiales eran muy reducidos, las escalas salariales tenían un diferencial pequeño, y además decreciente ${ }^{33}$. Por tanto el comportamiento de los trabajadores tendía a asegurar los pequeños pluses asociados a un sobrecumplimiento del plan ${ }^{34}$, pero no a ir más allá. Los managers estaban de acuerdo en esas prácticas, porque les permitían también a ellos obtener los pluses, bastante magros y no presionaban por un elevado nivel de eficiencia. De nuevo el problema de asimetría informativa hacía que el planificador no pudiese evaluar correctamente la ineficiencia productiva de la empresa, que era muy elevada. Los directivos de la misma sí podían evaluarla, pero sabían que no contaban con los instrumentos necesarios para reducirla de forma que dedicaban más esfuerzo a ocultar la información relevante que a otra cosa.

En general, el nivel de formación de los trabajadores era reducido, más allá de la formación inicial, y la movilidad también porque las diferencias salariales solo eran significativas en algunas zonas en las que los desincentivos ambientales eran muy fuertes. Además los trabajadores percibían que un incremento salarial en muchos casos no llevaba aparejado una mejora de su utilidad porque no había bienes en los que gastarlo.

La actividad económica de los trabajadores estaba así influenciada por el funcionamiento general de la sociedad en la que vivían. Determinados comportamientos resultaban favorecidos y otros desalentados y esto tenía mucha influencia en los resultados agregados de la economía soviética, como por ejemplo en la debilidad de la productividad, que sobre todo se hizo patente a partir de la década de los 70 . El efecto era más fuerte si tenemos en cuenta que la sociedad soviética, precisamente a consecuencia

${ }^{31}$ La planificación asignaba los trabajadores a las unidades de producción.

${ }^{32}$ Un buen análisis se puede encontrar en Lane (1987).

${ }^{33}$ Sólo se daban incentivos significativos a quienes decidiesen ir a trabajar a Siberia o al Círculo Polar Artico como explica Lane (1987).

${ }^{34}$ El sobrecumplimiento solía ser fácil de conseguir dada la baja utilización del capital y el bajo nivel de esfuerzo que implicaba el mero cumplimiento. Ver Lane (1987) o Nove (1977). 
de los planteamientos ideológicos del marxismo, había cambiado poco a lo largo del tiempo. La iniciativa individual, en todos los campos, era desalentada, de manera que no había espacio a más cambios que los decididos desde la cumbre de la planificación. Por tanto los agentes económicos llevaban décadas habituados a una serie de comportamientos y no otros. Desde al menos 1930, cuando se llevó a cabo la colectivización de la propiedad de la tierra, las instrucciones del sistema colectivista eran normas de obligado cumplimiento para la población.

En el momento de la transición, casi sesenta años después, no había agentes económicos que no hubiesen crecido dentro de la sociedad soviética. En cuanto a agentes económicos su comportamiento estaba conformado por décadas de sistema soviético. Los consumidores no conocían el auténtico significado de los precios como señales, ni estaban habituados a tomar decisiones sobre un conjunto amplio de alternativas. Los responsables de las empresas no tenían apenas en consideración a sus clientes o potenciales clientes ni sabían nada acerca de cómo vender un producto. Eran muy hábiles ocultando información, muchas veces con la connivencia de autoridades políticas regionales, y creando redes de trueque para proveerse de lo necesario para cumplir con sus objetivos. Son éstos los agentes económicos que realizan la transición económica en Rusia. El proceso implicaba una creación de mercados que como tales no existían y suponía que los mercados funcionarían bien. Pero no tenía lugar en el vacío, sino en una sociedad concreta en la que estaban insertos los comportamientos económicos de los agentes. Y esa cuestión no podía modificarse de la noche a la mañana.

\section{EL PROCESO DE TRANSICIÓN}

Desde comienzos de Abril de 1991 con la reducida liberalización de los precios puesta en marcha por Pavlov ${ }^{35}$ acompañada de la falta de control efectivo sobre los mismos de un Gosplan cuya existencia dejaba de tener sentido, la inflación se situó en niveles cercanos al $100 \%$ en tasa interanual y después siguió creciendo ${ }^{36}$. La inflación reprimida en la economía soviética saltó al primer plano, tal y cómo los expertos habían previsto. A finales del año 1991 y hasta la mitad de 1992 el gobierno dirigido por Gaidar controló de forma estricta la M2 y consiguió doblegar temporalmente la inflación ${ }^{37}$,

\footnotetext{
${ }^{35}$ Dabrowski (1997:43).

${ }^{36}$ Las cifras aparecen al final de la parte 2 del trabajo.

${ }^{37}$ Shleifer y Tresiman (2000).
} 
pero los continuos cambios de gobierno llevaron a la dirección del Banco Central a Viktor Gerashchenko, quien había sido una figura destacada en aspectos monetarios durante la época soviética. Inmediatamente comenzó una política de incremento de la masa monetaria para atender tanto a las necesidades de financiación del déficit público como del presupuesto regional y las demandas de las empresas. Eso incrementó la inflación durante varios años puesto que hasta finales de 1995 no se hicieron de nuevo intentos serios para estabilizar la economía.

Como explicamos en la parte 2 una medida fundamental para afianzar la transición económica era el control de la política monetaria y del déficit público. En lugar de hacer eso el Banco Central, con la anuencia de los gobiernos puso en marcha una política monetaria muy laxa que en algunos momentos llegó incluso a la monetización del déficit público. Gerashchenko estaba en estrecho contacto con destacados representantes del aparato de producción desde la época soviética y respondía a las peticiones de liquidez de todos ellos así como a las de los sectores que dependían para su existencia del presupuesto estatal.

La restructuración del aparato productivo de la época soviética para adaptarse a una situación de libre mercado requería reducir sustancialmente el peso del sector público en la economía y que las empresas se financiaran a partir de sus ventas y recurriendo al mercado. Pero eso era en teoría. En la práctica las empresas, que en 1991 eran todavía estatales y después se privatizaron, tenían unos lazos muy sólidos con los gestores de la política económica que salvo excepciones eran personas que habían ocupado puestos de responsabilidad en la época soviética. Esos lazos eran las conexiones de la red que los ligaban a todos ellos. Por tanto las decisiones de política económica que adoptaron no pueden aislarse de su pertenencia a esas redes. La supervivencia de muchas empresas y gobiernos regionales se conseguía al coste de la hiperinflación, al no acompañar una política monetaria drástica y el control del déficit a la liberalización de los precios. Al final del proceso los precios eran libres, como es propio en una economía de mercado pero la condición de acompañamiento necesaria para que esa economía de mercado funcionase bien, no se había cumplido. Y la razón residía sobre todo en el peso de las redes de contactos entre quienes tenían que tomar las impopulares decisiones y los dirigentes de las unidades económicas y políticas del país ${ }^{38}$. Esa era un rasgo heredado

${ }^{38}$ Es muy significativo que con el paso del tiempo los liberales hayan sido completamente desplazados del panorama político de Rusia. Han cargado con el peso de la responsabilidad por los problemas de la transición económica. 
del pasado pero que no podía suponerse que desaparecería con la eliminación de la economía colectivizada.

Las modificaciones sociales son en muchos casos muy lentas y en la medida en que la actividad económica y los comportamientos de los agentes se insertan en la realidad social, la influencia se mantiene. Una cosa, la más fácil, era tomar una medida en el sentido de liberalizar la economía, en este caso los precios. Otra más difícil era implementarlo de forma que produjese el efecto deseado. La última, que casi siempre está más allá del alcance de quien toma las decisiones implica la modificación de comportamientos que se insertan en la realidad social. Salvo que ésta se modifique rápida y drásticamente esos comportamientos se mantienen.

Lo anterior es todavía más claro cuando se analiza el proceso de privatización de las empresas. El gobierno de Yeltsin lo presentó en Marzo de 1992 y fue adoptado en Junio. A comienzos del año, antes de presentar, el plan el gobierno urgió las empresas a convertirse en compañías por acciones ${ }^{39}$ que debían venderse en el mercado ${ }^{40}$. El gobierno entregó bonos por valor nominal de 10.000 rublos a todos los rusos para que con ellos pudiesen comprar acciones de las empresas que se iban a privatizar, acudir a las subastas abiertas o revenderlos en un mercado abierto. Resultó muy significativo que la gran mayoría de los 144 millones de rusos a los que se les dieron los bonos los revendieran con carácter inmediato. El objetivo era crear una especie de capitalismo popular que repartiese la propiedad de las empresas entre los rusos, pero éstos lo rechazaron de plano. ¿Por qué no querían actuar como accionistas de empresas privadas? ¿Por qué rechazaron la oportunidad? Su comportamiento tan sólo se entiende cuando se examina a la luz de su inserción en una sociedad que había cambiado poco. En esa sociedad la propiedad privada tenía connotaciones negativas, siendo lo colectivo lo preferible. Además el cálculo económico había estado totalmente ausente de sus vidas. Apenas podía elegir como consumidores y los incentivos económicos como trabajadores eran poco relevantes. La condición de accionistas de una empresa privatizada estaba muy lejos de todo lo que había sido su comportamiento como agentes económicos hasta hacía muy poco tiempo, y eso no podía modificarse rápidamente. Por tanto la gran mayoría de ellos vendieron los bonos que fueron a parar a quienes sí tenían interés en hacerse con las acciones de las empresas.

\footnotetext{
${ }^{39}$ Algunos sectores como la industria de Defensa, y la producción de electricidad y gas quedaron fuera del proceso.

${ }^{40}$ Chubais y Vishneskaya (1997), Blasi et alii (1996).
} 
La segunda parte del programa consistía en ofrecer a los managers y trabajadores de las empresas una serie de opciones para que pudiesen comprar las acciones de las empresas en las que trabajaban suponiendo que eran los que más interés podían tener en ellas. Las opciones eran distintas ${ }^{41}$ pero lo fundamental eran dos cuestiones: la primera que los trabajadores y managers podían elegir por cual se decantaban, siempre la más favorable a sus posibilidades. La segunda, que quedaba oculta, era que un factor fundamental era el cálculo del valor de la empresa a privatizar. Un valor elevado implicaba que el gasto en efectivo para comprarla era grande y por tanto los interesados tenían dificultades para hacerse con la propiedad. Tanto los managers como los trabajadores, sobre todo los primeros, presionaron para que la valoración de las empresas se hiciese de acuerdo al valor en libros de los activos, que no se había actualizado en mucho tiempo porque no era necesario para el funcionamiento de la planificación. Dado que no había valores alternativos, no existía un precio de mercado en una economía en la que se estaban creando y que lo prioritario era privatizar la empresa rápidamente y no valorarla bien, ese valor es el que se utilizó.

El resultado es que en 1995 el 54,8\% de los propietarios de las empresas privatizadas eran «insiders» ${ }^{42}$, sean gerentes o trabajadores, y de los «outsiders», un total del $35,2 \%$, un $10,9 \%$ eran propietarios individuales, un $15,0 \%$ empresas financieras y tan sólo un 9,3\% recogía a las empresas financieras, holdings e inversores extranjeros. El resto seguía en manos del Sector Público. Los extranjeros fueron explícitamente excluidos del control de las empresas en la gran mayoría de los casos. Por un lado por el temor de las autoridades económicas a la pérdida del control del aparato productivo. Por otro lado por la presión de los interesados.

La importancia de este hecho es mayor de lo que parece. Los posibles compradores extranjeros estaban habituados a tomar decisiones y actuar dentro de economías de mercado y de sociedades en las que los mercados funcionaban con normalidad. Por lo tanto la manera en la que tomaban decisiones partía de su inserción en economías de mercado. En cambio en el caso de los propietarios rusos de las empresas privatizadas su comportamiento estaba influenciado por las redes sociales en las cuales estaban insertos, y que seguían siendo las de la época soviética aunque modificadas. La deriva de las empresas privatizadas rusas en el corto plazo se explica en gran medida por ese hecho. Al no permitir la entrada de propietarios extranjeros se cerró la posibilidad de

\footnotetext{
${ }^{41}$ Chubais y Vishnevskaya (1997: 71-73).

${ }^{42}$ Kapelyushnikov. R.I. (2000).
} 
que se incorporasen a la gestión comportamientos de mercado por parte de gestores acostumbrados al funcionamiento de los mismos.

Las autoridades regionales apoyaron el procedimiento.

The acquiescence of regional governments was bought by giving them control over small-scale privatization. Most shops and some small firms were sold by local governments for cash. In addition, regional property committees retained 15 to 20 percent of the shares of many enterprises to be privatized later ( Shleifer y Treisman 2000:31).

Hay que tener en cuenta que la distribución espacial de las empresas durante la época soviética entrañaba un fuerte nivel de especialización, de manera que en muchas ocasiones una única empresa, grande, era la fuente de rentas y empleo de un Oblast ${ }^{43}$ entero. Por tanto los lazos entre las autoridades políticas y los directivos de las empresas a nivel regional eran muy fuertes porque compartían intereses y objetivos. La red así creada era muy densa.

Además los nuevos bancos comerciales, que se financiaban casi siempre recurriendo al Banco Central, prestaron dinero a los antiguos managers de las empresas soviéticas para comprar su propia empresa en el proceso de privatización: una parte vía lo que les correspondía en el esquema de privatización adoptado, otra comprando bonos de los rusos que querían desprenderse de ellos y una última comprando acciones de muchos trabajadores a los que lo único que les interesaba era la disponibilidad de recursos y no tener acciones en su empresa. Las garantías para esos préstamos eran los propios activos de la empresa cuyas acciones se estaban comprando.

En conjunto, la mayoría de las empresas que se privatizaron fueron a parar sobre todo a los antiguos managers y directivos de las mismas o de otras. La población vendió los bonos de que disponían y las acciones que los trabajadores adquirieron en el proceso también se transfirieron con rapidez a ese grupo de personas. Las ventajas del proceso desde el punto de vista general eran obvias: por un lado se conseguía privatizar efectivamente y en un plazo breve la mayoría de las empresas ${ }^{44}$. Por otro lado se evitaba la entrada de extranjeros y la dependencia de ellos. En tercer lugar las empresas pasaban a estar gestionadas por personas expertas en la gestión en la época

${ }^{43}$ Un Oblast sería el equivalente a una de nuestras provincias, aunque había algunos muy extensos.

${ }^{44}$ Para 1997 se consideraba que el proceso estaba terminado, salvo en lo que hace a empresas que se decidió dejar bajo control público. 
soviética y que en general conocían bastante bien la firma y el entorno en el que se movía.

De esta manera se habían creado las empresas privadas imprescindibles para el funcionamiento de una economía de mercado. Pero su funcionamiento no se realizaba en el vacío, sino de acuerdo con las prácticas y comportamientos de quienes las regían. Los propietarios de las nuevas empresas privadas eran en su gran mayoría los managers y directivos de las antiguas firmas soviéticas. Llevaban con ellos las redes sociales que habían ido tejiendo en la época anterior y las formas de actuar. Eran muy expertos en determinadas cosas pero poco o nada en otras áreas porque la actividad económica en la época soviética se desarrollaba dentro de unos parámetros determinados. El funcionamiento de las nuevas empresas privadas en la economía rusa sería el resultado de la interacción de sus formas de actuar y de los cambios reales, que no teóricos, que se habían producido en su entorno.

Ya hemos señalado antes que la política monetaria no fue restrictiva y que el presupuesto presentaba grandes deficits como consecuencia de la decisión de no cortar la financiación a las regiones y a las empresas. Eso iba contra un pilar fundamental del cambio económico. Las empresas privatizadas debían dejar de depender de la financiación pública para acudir al mercado. Eso las obligaría a orientar su producción al mercado porque, si no lo hacían así, no podrían venderla y por tanto no podrían sobrevivir. En el fondo se trataba de obligar a las empresas a producir bienes y servicios con salida en el mercado, o a cerrar de forma que los recursos y los factores de producción pudiesen utilizarse en otros sectores.

El mantenimiento de la financiación con cargo al presupuesto les evitaba enfrentarse a esa disyuntiva. Los nuevos propietarios de las empresas en su gran mayoría decidieron seguir haciendo lo que sabían hacer, producir bienes y servicios con el mismo equipo de capital preexistente y en gran medida con la misma mano de obra. Parte de esa producción podía venderse en el nuevo mercado libre, tanto en el interior como en el extranjero ${ }^{45}$, pero otra parte no se vendía. La subsiguiente acumulación de stocks y la falta de liquidez sólo se podían evitar gracias a las inyecciones financieras que venían desde el presupuesto.

En este punto entraban en juego las redes sociales que se habían creado durante la época comunista. Gran parte de los empresarios rusos eran antiguos dirigentes de empresas soviéticas. Buena parte de los políticos de la Federación Rusa y de los niveles

${ }^{45}$ Fundamentalmente materias primas energéticas y metálicas. 
locales eran también antiguos altos miembros del PCUS ${ }^{46}$. Las redes creadas durante la época soviética se mantuvieron y son las que explican que los nuevos empresarios consiguiesen que el presupuesto siguiese financiando parcialmente las empresas. Los políticos locales participaban de esas redes y tenían un interés particular en que las empresas siguiesen funcionando dado que en muchas zonas eran la principal fuente de empleo y rentas. La sociedad rusa no era igual que la sociedad soviética, pero en algunos aspectos el cambio no había sido muy profundo. La actuación de los agentes económicos en la Rusia de los 90 seguía inserta en unas estructuras y unas redes que habían comenzado en la época soviética, pero que no habían desaparecido. El cambio político y económico no había alterado significativamente esos hechos, en gran medida porque los actores principales del mismo eran parte integrante de las redes y no tenían interés alguno en eliminarlas, sino en utilizarlas en su provecho en la nueva situación. Es más, desde la posición que ocupaban en esas redes habían influido para que el cambio les afectase en la menor medida posible e intentaron con éxito en muchas ocasiones configurarlo de la forma más favorable a sus intereses ${ }^{47}$.

A pesar del recurso a la financiación presupuestaria las empresas se encontraron con un gran problema de liquidez porque parte de su producción no podía venderse. El problema era muy general y afectaba a muchas empresas que estaban en la misma situación. La única solución fue recurrir al trueque. El pago de parte de los bienes y servicios sólo podía hacerse entre las empresas intercambiándolos por otros bienes. Pero eso era precisamente lo que las redes de la época soviética, la Blat sabían hacer muy bien. Las redes continuaban existiendo porque estaban compuestas de lazos personales y podían seguir solucionando un problema de falta de liquidez recurriendo al trueque al que estaban tan acostumbrados sus miembros. En este sentido, la antigua red de época soviética fue parte de la solución del problema, porque sin ella las dificultades hubiesen sido peores, pero al mismo tiempo era uno de los elementos que generaba las

${ }^{46}$ Conforme la situación política en la URSS se deterioraba a finales de los 80, buena parte de los dirigentes del partido, del estado y de las repúblicas se reconvirtieron primero en liberales y después en nacionalistas para mantenerse en los aledaños del poder. Es significativo al respecto por un lado el escasísimo apoyo que tuvo el golpe de estado de Agosto de 1991 y por otro que el Partido Comunista de Gennadi Ziuganov apenas contase entre sus miembros con miembros relevantes del antiguo PCUS.

${ }^{47}$ Algunos procesos de privatización en localidades pequeñas no pueden entenderse sin tener en cuenta la connivencia entre las autoridades políticas y los directivos de las empresas colectivizadas que diseñaron mecanismos a la medida y excluyeron, por cualquier medio, la posibilidad de que otros agentes tuviesen opciones. Ver Shleifer y Treisman (2000) 
distorsiones. En un mercado «normal» en el que tal tipo de redes no existen por un lado el trueque es mucho más difícil, lo que hubiese puesto en apuros a muchas empresas. Pero por otro no hubiesen podido presionar para que las empresas que producían bienes y servicios que tenían poca demanda recibiesen financiación del presupuesto.

El objetivo final de la transición económica rusa era conseguir una restructuración de la economía para orientarla a las necesidades y gustos de los consumidores y hacerla más productiva. Para ello era necesario, de alguna manera, obligar a las empresas, con los incentivos apropiados a cambiar su orientación. Eso no se hizo porque los propietarios de las empresas a través de las redes creadas en la época soviética consiguieron evitar la presión de esos incentivos. Las redes configuraron la manera en la que se realizó la transición porque actuaron en la forma en la que estaban habituadas a hacerlo. Los mercados no funcionaron de la manera en la que se suponía que tenían que hacerlo porque los principales agentes de los mismos, que eran ya agentes significativos en la época soviética del no-mercado, estaban insertos en un conjunto de redes que utilizaron para configurar el comportamiento del mercado. La densidad de esas redes previas y el hecho de que el proceso de privatización excluyera conscientemente a agentes extranjeros que no pertenecían a las mismas les permitió manejarlo a su antojo. Las empresas eran privadas, actuaban en mercados libres pero no de la forma que» a priori» se esperaba de ellas.

Los problemas de liquidez afectaron también a los salarios de los trabajadores, pero apenas a sus puestos de trabajo. Como hemos puesto de manifiesto en la segunda parte del trabajo el incremento del desempleo fue muy inferior al esperado. La clave residió en que buena parte de las antiguas empresas soviéticas continuaron produciendo como empresas privadas gracias a lo que hemos comentado más arriba. Una de las razones por las que las autoridades locales de la Federación de Rusia apoyaron a las empresas privatizadas era que constituían en muchos casos la casi única fuente de empleo y renta y un desempleo masivo les hubiera acarreado muchos problemas en el plano social y político. De forma indirecta la financiación estatal de las empresas servía para mantener el empleo. A cambio los trabajadores tuvieron que aceptar retrasos y reducciones reales en los pagos de los salarios porque las empresas vendían menos de lo que producían. Sus alternativas no eran mejores. Su nivel de especialización y capital humano era reducido, habían trabajado durante muchos años en un solo sector o empresa y les resultaba muy difícil cambiar. Durante los primeros años, además, no surgieron nuevos sectores productivos a los que dedicarse.

En conjunto la economía rusa no se reestructuró. La producción disminuyó mucho, como hemos puesto de manifiesto, debido a que en gran medida no se reorientó hacia la demanda, pero muchas empresas no cerraron ni cambiaron radicalmente de 
dirección y estrategia porque no les fue necesario. Como consecuencia el desempleo creció pero en ningún caso fue masivo. La financiación por parte del presupuesto de empresas privatizadas y de las regiones exacerbó la inflación, mucho más cuando parte del déficit público se monetizó. El tipo de cambio del rublo se deterioró de forma continua. Eso abocó a la economía rusa a la gran crisis de 1998. Resulta paradójico pensar que todos los expertos, tanto rusos como extranjeros, predecían una gran crisis (la transformación) al comienzo de la transición, seguida de una recuperación hasta colocar a Rusia como una economía moderna y boyante. En lugar de eso los datos muestran un proceso de deterioro continuo que toca fondo en 1998 y después remonta.

\section{Conclusiones}

En este trabajo hemos puesto de manifiesto la importancia de la inserción de los agentes económicos en la sociedad para explicar lo ocurrido durante la transición económica en Rusia. En primer lugar los agentes económicos durante la transición económica han sido casi los mismos que durante el final de la época soviética, y desempeñando papeles muy parecidos. Eso es debido por un lado a que de forma deliberada se excluyó a otros agentes, sobre todo extranjeros. Por otro lado a que no había en Rusia a comienzos de los años 90 personas capaces de gestionar una empresa privada que no fuesen los antiguos gerentes de las empresas colectivas. Todos esos agentes habían actuado durante años insertos en una realidad social poco móvil. Por su naturaleza la sociedad soviética se modificaba menos y más despacio que una sociedad de economía de mercado. Por consiguiente su comportamiento como empresarios, trabajadores o consumidores había seguido durante mucho tiempo unas pautas que no desaparecieron en la transición. La realidad social comenzó a cambiar más deprisa, porque la libertad lo facilita, pero no de forma inmediata y los agentes se comportaron tal y cómo estaban acostumbrados a hacerlo, al menos en parte. Como consecuencia consiguieron influir en el rumbo de la transición económica y eludir la reestructuración de la economía. El resultado fue la enorme reducción del PIB, la inflación y un nivel aceptable del desempleo. El crecimiento de la masa monetaria fomentado por el gobierno para varios fines se combinó con lo anterior para producir la crisis general de 1998.

En el caso de Rusia la importancia de la inserción en la sociedad es más fuerte porque la ideología comunista lo imponía. Al suponer que la base material- económicadeterminaba los comportamientos a todos los niveles de las personas el aparato institucional comunista delimitó de forma muy estricta la actuación de los agentes en el ámbito económico. Por esa razón el caso de la transición económica en Rusia resulta tan 
significativo para estudiar esta cuestión. Eso mismo debe llevarnos a tomar una cierta perspectiva al aplicar el análisis a otras sociedades. En sociedades más abiertas, los agentes son libres para tomar sus propias decisiones, por un lado, y por otro las sociedades les constriñen mucho menos, de forma que no puede suponerse que todo lo que hacen esté socialmente determinado. Es necesario estudiar hasta qué punto están los comportamientos económicos insertos en cada sociedad concreta. Pero eso no elimina la necesidad de analizarlo. Las redes sociales son un componente fundamental en el comportamiento económico de los agentes ${ }^{48}$. Generan unas pautas y unas expectativas que sirven de referencia a sus miembros, a la vez que constituyen el caldo de cultivo básico de muchas relaciones entre empresas que se supone que se rigen exclusivamente por criterios de mercado impersonal ${ }^{49}$.

Una enseñanza muy clara del caso ruso es que la transición económica no destruyó esas redes. Tampoco lo intentó. Lo que ocurrió es que las redes (la Blat) eran tan densas y tenían tanta historia detrás en la Unión Soviética que consiguieron darle una forma concreta a la transición y la desviaron del objetivo último de la misma. Cuando se analiza a fondo el proceso de privatización en Rusia se observa cómo desde Moscú emanan las directrices fundamentales, primero, y después los procedimientos concretos. Cuando las directrices llegan a un Oblast, por ejemplo en Siberia, las autoridades políticas y los dirigentes de las empresas las aceptan, pero ponen todas las trabas posibles a los procedimientos y lo paralizan. En su lugar esas redes locales proponen modificaciones de calado a los procedimientos para garantizar el cumplimiento de las directrices. El gobierno en Moscú acepta esas modificaciones, la privatización se lleva a cabo pero en las condiciones que la red local quiere y que le benefician. Las redes son resistentes porque involucran a muchos agentes y tienen un pasado de relaciones que favorece el funcionamiento del sistema. Con menor intensidad que en el caso ruso, y variable en cada ocasión, las redes en una economía de mercado presentan el mismo comportamiento, favorecen determinados comportamientos colectivos y desalientan otros. De forma más general las preferencias de los agentes económicos resultan influenciadas

${ }^{48}$ Es muy interesante el análisis que realiza Abolafia (1996) sobre los comportamientos de los agentes en los mercados de Wall Street. El autor muestra que lo que puede y debe hacerse, así como lo que no puede ni debe hacerse en ellos tiene mucho que ver con las redes en las que los agentes se insertan.

${ }^{49}$ Es bien sabido que las Escuelas de Negocio o las Universidades más prestigiosas dan lugar a un entramado de relaciones que se mantienen a lo largo de la carrera profesional de muchos de sus alumnos y de esa manera influyen en las decisiones de las empresas que ellos rigen. 
por los comportamientos colectivos insertos en la sociedad en la que viven ${ }^{50}$. Y eso ha de ser tenido en cuenta. Lo primero, desde luego, es establecer la fuerza relativa de esa influencia, que no tiene porqué ser igual en todas partes. Después hay que utilizarla para ayudar a explicar los fenómenos ${ }^{51}$.

El examen de la transición económica también pone de manifiesto que muchas veces las limitaciones cognitivas ${ }^{52}$ juegan un papel relevante en la toma de decisiones. Esto es perfectamente conocido pero se ha subrayado poco que, ante limitaciones cognitivas, los agentes pueden basar sus decisiones en los comportamientos de su medio social. La decisión de los rusos de desprenderse de los bonos con los que podían haber comprado bonos de empresa privatizadas parte de problemas cognitivos porque en muchos casos no eran capaces de comprender lo que implicaban los bonos. En esa circunstancia actúan de acuerdo con lo que el medio social en el que viven hace. Una actitud mucho más «racional» hubiera sido mantenerlos y obtener de ellos los dividendos correspondientes. Por ese medio el gobierno ruso hubiese conseguido el objetivo de hacer a los rusos partícipes de la propiedad de las empresas e involucrar a un gran porcentaje de la población en el proceso. Posiblemente era eso lo que el gobierno esperaba y en ese sentido su decisión era la correcta, pero en otro medio social, no en el que había en la Rusia post-comunista de principios de los años 90. Los destinatarios de la medida no estaban listos para ella ${ }^{53}$.

El análisis económico parte del planteamiento de la eficiencia y el equilibrio en los mercados y sólo cuando tropieza con dificultades explicativas introduce varios tipos de ineficiencia para que las desviaciones entre lo que se supone que debería pasar y lo que ocurre desaparezcan o se reduzcan mucho. Ese es un planteamiento correcto mientras no sea sistemático, porque en estos casos la ventaja de la simplicidad de planteamiento

${ }^{50}$ Los expertos señalan siempre que en España hay una preferencia «social» por la vivienda en propiedad que resulta difícil racionalizar en términos de criterios meramente «económicos»

${ }^{51}$ En un caso diferente, la economía China, se ha señalado el dinamismo en la producción y creación de empleo de pequeñas y medianas empresas que son propiedad de comunidades locales. No está bien determinado a quien pertenece la propiedad y tampoco la toma de decisiones pero en muchas ocasiones funcionan igual o mejor que las empresas privadas y siguen estrategias parecidas. Su base es un cierto «comunalismo» en la propiedad de los recursos y del trabajo.

52 «Bounded Rationality» H.A.Simon.

${ }^{53}$ No todos, desde luego. Había grupos de agentes que conocían perfectamente las ventajas de adquirir porcentajes significativos de acciones y lo hicieron. Por tanto no puede decirse que el entorno social en el que están insertos los agentes «determina»su comportamiento porque no es verdad. Pero que lo influye es indudable. 
inicial se pierde. Un buen acercamiento en muchos casos puede ser tener en cuenta hasta qué punto los comportamientos de los agentes dependen de su inserción en un medio social dado y las redes que se generan en él. De esta forma se pierde algo de la generalidad del análisis pero se gana en precisión. La elección depende, desde luego, de cuál es la importancia de la generalidad y la precisión en cada uno de los casos. En el caso de la transición rusa hemos mostrado que la atención a esa inserción ayuda mucho a entender los fenómenos que se dieron.

\section{REFERENCIAS}

Abolafia, M. (1996), Making Markets. Opportunism and Restraint on Wall Street, Cambridge (MA), Harvard University Press.

Abolafia, M.; Biggart, Y. y Woolsey, N. (1991), «Competition and Markets. An Institutional Perspective», en A. Etzioni y P. R. Lawrence (1991), Socio-Economics. Toward a New Synthesis, Londres, Armonk, págs. 211-231.

BERLINER, J. S. (1957), Factory and Manager in the USSR, Cambridge (MA), Harvard University Press.

Blasi, J.; Kroumova, J. y Shleifer, A. (1996), Kremlin Capitalism. Privatizing Russia, Cornell, Cornell University Press.

Chubais, A. y Vishnevskaya, M. (1997), «Main Issues of Privatization in Russia», en A. Aslund (ed.), Russia's Economic Transformation in the 1990's, Londres, Pinter.

Dabrowski, M. (1997), «The First Half-Year of Russian Transformation», en A. Aslund (ed.), Russia's Economic Transformation in the 1990's, Londres, Pinter.

Doвв, M. (1960), Soviet Economic Development Since 1917, Londres, Routledge \& Kegan Paul.

Etzioni, A. (1999), Essays in Socio-Economics, Nueva York, Springer.

Granick, D. (1960), The Red Executive. A Study of the Organization Man in Russian Industry, Nueva York, Doubleday.

- (1954), Management of the industrial firm in the USSR, Nueva York, Columbia University Press.

Granovetter, M. (2005), «The impact of Social Structure on Economic Outcomes», Journal of Economic Perspectives, 19-1, págs. 33-50.

- (1990), «The Old and the New Economic Sociology», en R. Friedland y A. F. Robertson, Beyond the Market Place, Nueva York, Walter de Gruyter.

- (2005), (1985), «Economic Action and Social Structure: The Problem of Embeddedness», American Journal of Sociology, 91-3, págs. 481-510. 
Granovetter, M. y Swedberg, R. (2001), The Sociology of Economic Life, Boulder (Col.), Westview Press.

Gregory, P. y Stuart, R. (2001), Russian and Soviet Economic Performance and Structure, Nueva York, Addison Wesley.

Kapelyushnikov, R. I. (2000), «The Largest and Dominant Shareholders in the Russian Industry: Evidence of the Russian Economic Barometer Monitoring», Russian Economic Barometer, 9 (1), págs. 9-46.

LANe, D. (1987), Soviet Labour and the Ethic of Communism, Boulder (Col.), Westview Press.

- (1985), Soviet Economy and Society, Nueva York, New York U. Press.

Ledeneva, A. (1998), Russia,s Economy of Favours. Blat, Networking and Informal Exchange, Cambridge (UK), Cambridge University Press.

Lipton, D. y SACHS, J. (1990), «Creating a Market Economy in Eastern Europe: The Case of Poland», Brooking Papers on Economic Activity, 1, págs. 75-147.

Nove, A. (1969), An Economic History of the USSR, Londres, Harmondsworth.

- (1977), The Soviet Economic System, Londres, George Allen \& Unwin.

Polanyi, K. (1944), The Great Transformation, Nueva York, Holt.

Polanyi, K.; Arensberg, C. y Pearson, H. (1957), Trade and Market in the Early Empires, Nueva York, Free Press.

Roland, G. (2000), Transition and Economics. Politics, Markets and Firms, Cambridge, MIT Press.

Shleifer, A. y Treisman, D. (2000), Without a Map. Political Tactics and Economic Reform in Russia, Cambridge (MA), Mit Press.

Simon, H. A. (1982), Models of Bounded Rationality, vols. 1 y 2. Cambridge (MA), MIT Press.

Swedberg, R. y Granovetter, M. (1992), «Introduction», en R. Swedberg y M. Granovetter (eds.), The sociology of Economic Life, Londres, Westview Press. 